\title{
BMJ Open Association of proton pump inhibitors and concomitant drugs with risk of acute kidney injury: a nested case-control study
}

\author{
Keiko Ikuta, ${ }^{1}$ Shunsaku Nakagawa (D) , ${ }^{1}$ Kenji Momo, ${ }^{2}$ Atsushi Yonezawa (D) ,1,3 \\ Kotaro Itohara, ${ }^{1}$ Yuki Sato, ${ }^{1}$ Satoshi Imai, ${ }^{1}$ Takayuki Nakagawa, ${ }^{1}$ \\ Kazuo Matsubara ${ }^{1,4}$
}

To cite: Ikuta K, Nakagawa S, Momo K, et al. Association of proton pump inhibitors and concomitant drugs with risk of acute kidney injury: a nested case-control study. BMJ Open 2021;11:e041543. doi:10.1136/ bmjopen-2020-041543

- Prepublication history and additional materials for this paper is available online. To view these files, please visit the journal online (http://dx.doi. org/10.1136/bmjopen-2020041543).

Received 11 June 2020 Revised 20 December 2020 Accepted 28 January 2021

Check for updates

(C) Author(s) (or their employer(s)) 2021. Re-use permitted under CC BY-NC. No commercial re-use. See rights and permissions. Published by BMJ.

${ }^{1}$ Department of Clinical Pharmacology and Therapeutics, Kyoto University Hospital, Kyoto, Japan

${ }^{2}$ Department of Hospital Pharmaceutics, School of Pharmacy, Showa University, Tokyo, Japan

${ }^{3}$ Graduate School of Faculty of Pharmaceutical Science, Kyoto University, Kyoto, Japan ${ }^{4}$ Department of Pharmacy, Wakayama Medical University, Wakayama, Japan

Correspondence to Dr Shunsaku Nakagawa; nakashun@kuhp.kyoto-u.ac.jp

\section{ABSTRACT}

Objectives This study aimed to assess whether the combined use of proton pump inhibitors (PPIs) with non-steroidal anti-inflammatory drugs (NSAIDs) or antibiotics (penicillins, macrolides, cephalosporins or fluoroquinolones) was associated with an increased risk of acute kidney injury (AKI).

Design A nested case-control study.

Setting A health insurance claims database constructed by the Japan Medical Data Center.

Participants Patients were eligible if they were prescribed a PPI, NSAID and antibiotic at least once between January 2005 and June 2017. The patients who were new PPI users and did not have any history of renal diseases before cohort entry were included $(n=219082)$. The mean age was 45 and $44 \%$ were women.

Interventions Current use of PPIs, NSAIDs, or antibiotics. Primary outcome measures Acute kidney injury. Results During a mean follow-up of 2.4 (SD, 1.7) years, 317 cases of AKI were identified (incidence rate of 6.1/10 000 person-years). The current use of PPIs was associated with a higher risk of AKI compared with past PPI use (unadjusted OR, 4.09; $95 \% \mathrm{Cl}, 3.09$ to 5.44). The unadjusted ORs of AKI for the current use of PPIs with NSAIDs, cephalosporins and fluoroquinolones, compared with the current use of PPIs alone, were 3.92 (95\% Cl, 2.40 to 6.52), 2.57 (1.43 to 4.62) and 3.08 (1.50 to 6.38$)$, respectively. The effects of concurrent use of PPIs with NSAIDs, cephalosporins or fluoroquinolones remain significant in the adjusted model. The analyses on absolute risk of AKI confirmed the results from the nested case-control study.

Conclusions Concomitant use of NSAIDs with PPIs significantly increased the risk for AKI. Moreover, the results suggested that concomitant use of cephalosporins or fluoroquinolones with PPIs was associated with increased risk of incident AKI.

\section{INTRODUCTION}

Previous studies have shown a possible association between the use of proton pump inhibitors (PPIs) and the increased risks of acute kidney injury (AKI), acute tubulointerstitial nephritis (AIN) or chronic kidney disease. ${ }^{12}$ Particularly, the interrelation between the use of PPIs and the pathogenesis of AKI has been

\section{Strengths and limitations of this study}

- This is the first study to investigate the association between concomitant use of non-steroidal antiinflammatory drugs (NSAIDs) or antibiotics with proton pump inhibitors (PPIs) and the risk of acute kidney injury among patients who were first-time or restarting PPI users.

- We used a health insurance claims database that enabled us to track data for each patient, even if the patient visited multiple medical institutions.

- The severity of acute kidney injury could not be evaluated because the database did not include serum creatinine level and glomerular filtration rate.

- The patients in this study were relatively younger than those in previous studies.

- The number of identified cases who concomitantly used NSAIDs or antibiotics with PPIs was relatively small.

suggested in several large-scale observational studies. ${ }^{3-10}$ Recently, it has been reported that the use of PPIs is an independent risk factor of AKI in patients administered with immune checkpoint inhibitors. ${ }^{11} 12$ This finding has highlighted a notion that concomitant drugs affect the risk of AKI in PPI users. PPI is often co-prescribed with potentially nephrotoxic drugs, such as non-steroidal anti-inflammatory drugs (NSAIDs) and antibiotics. However, the effect of concomitant drugs on the risk of adverse renal outcome in PPI users has been less investigated. Two studies have assessed risks of AKI when NSAIDs were concomitantly used with PPIs. ${ }^{1013}$ Although the results suggested that NSAIDs did not affect the risk of AKI in PPI users, these studies were limited by their insufficient statistical power ${ }^{10}$ and possible selection bias. ${ }^{13}$ Moreover, no study has evaluated the effect of concomitant use of antibiotics with PPIs.

Based on these backgrounds, we conducted a nested case-control study to determine whether the concomitant use of NSAIDs or 
antibiotics with PPIs was associated with an increased risk of AKI. This study was designed on the assumption that PPI-related AKI might develop rapidly and that current PPI use was associated with an increased risk of AKI. In contrast, previous studies have not considered the duration between the end of PPI use and the onset of $\mathrm{AKI}^{3-7}$ or assessed the risk of PPI-related AKI with relatively wide risk windows of 90 or 120 days. $^{8-10}$ Therefore, we further evaluated a relative risk of AKI for current PPI use in comparison with past use.

\section{METHODS}

\section{Data source}

We used a health insurance claims database constructed by the Japan Medical Data Center (JMDC) Co Ltd, Tokyo, Japan. The JMDC database is based on monthly medical claims submitted to health insurance societies from hospitals and community pharmacies in Japan since January 2005, and covers approximately 4 million corporate employees and their families. Personal identifier, age, gender, medical procedures, diagnostic codes using the International Classification of Diseases $10^{\text {th }}$ version (ICD$10)$ in the data were encrypted. This database consisted of brand name, class according to the Anatomical Therapeutic Chemical Classification System (ATC index), total number of units, daily dose and medication days of prescribed drugs. The database has been widely used in pharmacoepidemiological studies. ${ }^{14-16}$

\section{Study cohort}

The patients were eligible if they were prescribed a PPI, NSAID and antibiotic at least once between January 2005 and June 2017. The patients were excluded if they had incomplete information on data of prescribed PPIs, NSAIDs, penicillins, macrolides, cephalosporins and fluoroquinolones. Additionally, the patients were excluded if their medical histories before the first prescribing of PPIs were less than 6 months. The cohort entry was defined as the date when PPIs were prescribed for the first time. Finally, we excluded the patients who had history of renal dysfunction before the cohort entry. The definitions of renal diseases excluded from this study are shown in online supplemental table 1.

\section{Cases and controls}

The primary outcome was the development of AKI. We identified all patients who were potentially diagnosed with AKI after the cohort entry using the ICD-10 diagnostic codes (N17X). A previous study had shown that the ICD-10 code N17X for AKI has a moderate sensitivity and high specificity. ${ }^{17}$ The diagnosis date of AKI was defined as the index date for each case. The patients were excluded from cases if they had any episode of other renal diseases before the index date and if they were diagnosed with pyelonephritis or contrast-induced nephropathy at the index date. Up to 10 controls were randomly selected and matched to each case on the birth year ( \pm 1 year), gender and follow-up period ( \pm 180 days). The date of the risk set was the index date for the controls.

\section{Measurement of exposure}

The drugs of interest were PPIs (lansoprazole, esomeprazole, rabeprazole, omeprazole and vonoprazan), NSAIDs $^{18}{ }^{19}$ and four classes of antibiotics (penicillins, ${ }^{2021}$ macrolides, ${ }^{22} 23$ cephalosporins ${ }^{20}{ }^{21}$ and fluoroquinolones). ${ }^{24}$ We considered class effects of these drugs, regardless of daily dose or formulation types excluding topical agents. The ATC index codes to identify the study drugs are shown in online supplemental table 2. We divided the number of prescribed units by the daily dose to estimate the duration of drug use. In a previous nested case-control study that evaluated the effect of PPI use on the development of AIN, it has been shown that the use of PPIs within 30 days before the index date, compared with the other exposure status, was associated with an increased risk of AIN. ${ }^{25}$ Therefore, the exposure status was classified into three categories: current use, the drug use within 30 days before the index date; recent use, the drug use within 90 days, but not within 30 days, before the index date; and past use, the drug use after the cohort entry, but not within 90 days before the index date.

\section{Potential confounding}

In addition to matching factors, we assessed confounders, which were current use of nephrotoxic drugs, comorbidity and Charlson comorbidity index (CCI). The nephrotoxic drugs were selected from the drugs available in Japan, according to the drug formulary described in 'the
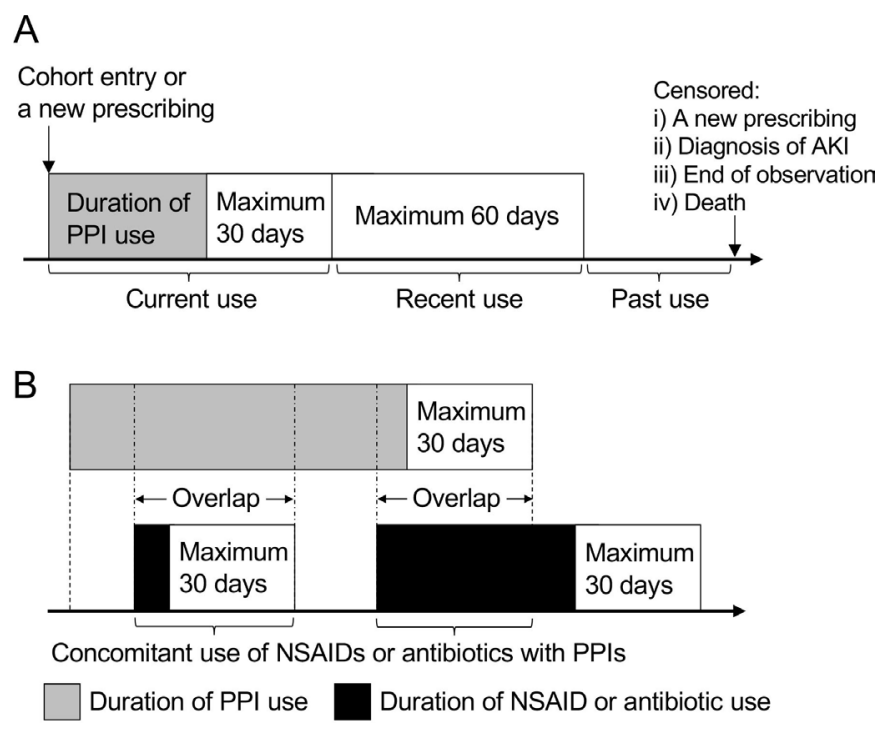

Figure 1 Determination of person-years of exposure. (A) Total person-years of each patient were divided into three categories: current use of PPIs, recent use of PPIs and past use of PPIs. (B) The duration of concomitant use of NSAIDs or antibiotics with PPIs was defined as the amount of time when the current use of PPIs overlapped with the current use of NSAIDs or antibiotics. AKI,acute kidney injury; NSAIDs, non-steroidalanti-inflammatory drugs; PPIs, proton pump inhibitors. 


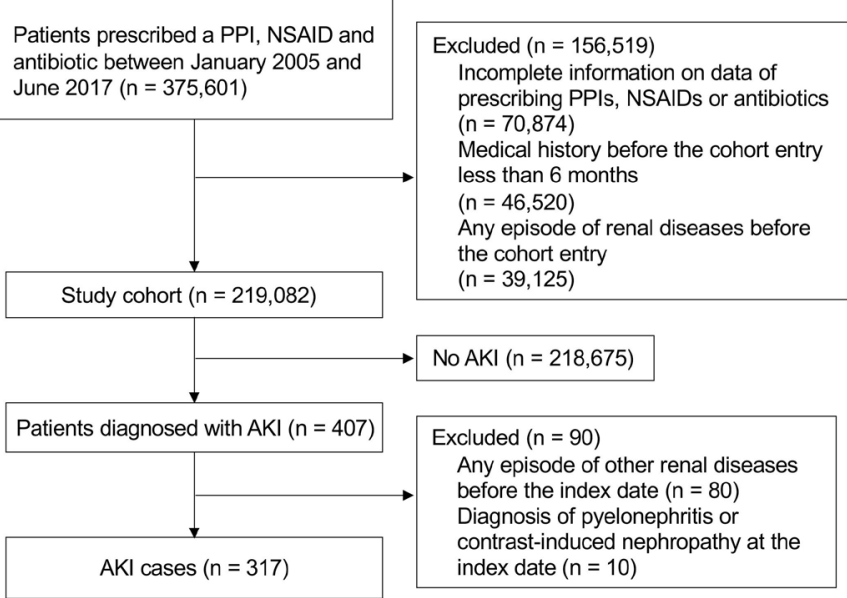

Figure 2 Flow diagram of cohort and case selection process. AKI,acute kidney injury; NSAIDs, non-steroidalantiinflammatory drugs; PPIs, proton pump inhibitors.

Clinical Practice Guideline for Drug-induced Kidney Injury in Japan 2016' published by the Japanese Society of Nephrology (online supplemental table 3). We used the ATC index codes or the specific names to identify the drugs suspected to increase the risk of AKI. We assumed that comorbidity influences exposure to study drugs and the risk of AKI and its detection. Since a correlation between higher CCI and the AKI has been shown, ${ }^{26-28}$ we used CCI as an indicator of comorbidities. We identified records of comorbidities (peptic ulcer, liver disease, congestive heart failure, cerebrovascular disease, peripheral vascular disease, myocardial infarction, diabetes, pulmonary disease, connective tissue disorder, cancer and renal disease) within 6 months before the index date. The classification of diseases using the ICD-10 codes and the calculation of CCI were performed according to previous reports. ${ }^{29}{ }^{30}$ Moreover, we identified patients diagnosed with hypertension using the ICD-10 codes of I10X.

\section{Person-years of exposure}

We determined person-years at risk for drug use by summarising the dispensing data (figure 1A), in accordance with the method described in a previous report. ${ }^{25}$ We divided the study period into three categories: current use (durations of drug use and 1-30 days thereafter), recent use (31-90 days after the end of drug use) and past use (more than 90 days after the end of drug use). All episodes were censored if a new prescription was started or the patients reached the end of observation, died or were diagnosed with AKI. The durations of all episodes of current and recent PPI use were summed to determine the person-years of current and recent PPI uses, respectively. We estimated the person-years of past PPI use by subtracting those of current and recent use from the total person-years of the study period. Furthermore, we calculated the person-years for drug combinations (figure 1B). The duration of concomitant use of NSAIDs or antibiotics with PPIs was defined as the amount of time when the
Table 1 Characteristics of cases and matched controls at the index date

\begin{tabular}{|c|c|c|}
\hline Characteristics & $\begin{array}{l}\text { Cases } \\
(n=317)\end{array}$ & $\begin{array}{l}\text { Controls } \\
(n=3150)\end{array}$ \\
\hline Age, years, mean (SD) & $52(13)$ & $52(13)$ \\
\hline Female, n (\%) & $117(36.9)$ & 1161 (36.9) \\
\hline $\begin{array}{l}\text { Duration of follow-up, days, } \\
\text { mean (SD) }\end{array}$ & 478 (512) & 487 (483) \\
\hline \multicolumn{3}{|l|}{ Comorbidity, n (\%) } \\
\hline Hypertension & $71(22.4)$ & 707 (22.4) \\
\hline Congestive heart failure & $20(6.3)$ & $84(2.7)$ \\
\hline Diabetes & $52(16.4)$ & $331(10.5)$ \\
\hline Liver disease & $31(9.8)$ & $230(7.3)$ \\
\hline Pulmonary disease & $20(6.3)$ & $183(5.8)$ \\
\hline Cancer & $56(17.7)$ & $132(4.2)$ \\
\hline $\begin{array}{l}\text { Charlson comorbidity index, } \\
\text { median (IQR) }\end{array}$ & 0 (0 to 2$)$ & 0 (0 to 1$)$ \\
\hline \multicolumn{3}{|c|}{ PPIs prescribed at the last time, $\mathrm{n}(\%)$} \\
\hline Lansoprazole & $118(37.2)$ & $1148(36.4)$ \\
\hline Esomeprazole & $86(27.1)$ & $758(24.1)$ \\
\hline Rabeprazole & $70(22.1)$ & $737(23.4)$ \\
\hline Omeprazole & $25(7.9)$ & $249(7.9)$ \\
\hline Vonoprazan & $18(5.7)$ & $258(8.2)$ \\
\hline $\begin{array}{l}\text { Current use of nephrotoxic } \\
\text { drugs, } \mathrm{n}(\%)^{\star}\end{array}$ & $199(62.8)$ & 1000 (31.8) \\
\hline Current use of NSAIDs, n (\%) & $87(27.4)$ & $297(9.4)$ \\
\hline Current use of penicillins, $n(\%)$ & $24(7.6)$ & $84(2.7)$ \\
\hline Current use of macrolides, $\mathrm{n}(\%)$ & $20(6.3)$ & $157(5.0)$ \\
\hline $\begin{array}{l}\text { Current use of cephalosporins, } \\
n(\%)\end{array}$ & 43 (13.6) & $149(4.7)$ \\
\hline $\begin{array}{l}\text { Current use of fluoroquinolones, } \\
n(\%)\end{array}$ & $26(8.2)$ & $94(3.0)$ \\
\hline
\end{tabular}

*Four cases (1.3\%) had missing data.

NSAIDs, non-steroidal anti-inflammatory drugs; PPIs, proton pump inhibitors.

current PPI use overlapped with the current NSAID or antibiotic use.

\section{Statistical analysis}

Since all available cases in the database were included, and the study sample size was governed by the disease incidence, a formal power calculation was not performed. All statistical analyses were performed using JMP software V.14 (SAS Institute Inc, Cary, North Carolina). A conditional logistic regression model was used to compute an OR and 95\% CI, which, for the nested case-control study, provides unbiassed estimates of the rate ratio. ${ }^{31}$ An adjusted OR was estimated by entering the potential confounders (current use of nephrotoxic drugs and CCI) into the model. To estimate the effect of concomitant drugs, we studied the influence of concomitant 
Table 2 Effect of proton pump inhibitor (PPI) use on the risk of acute kidney injury (AKI)

\begin{tabular}{lllll} 
Exposure of PPIs & Cases (\%), $\mathbf{n = 3 1 7}$ & Controls (\%), $\mathbf{n = 3 1 5 0}$ & OR (95\% Cl) & OR (95\% Cl), adjusted† \\
\hline Current use & $148(46.7)$ & $655(20.8)$ & $4.09(3.09$ to 5.44$)$ & $2.79(2.06$ to 3.79$)$ \\
Recent use & $23(7.3)$ & $416(13.2)$ & $1.26(0.72$ to 2.13$)$ & $1.02(0.57$ to 1.76$)$ \\
Past use & $146(46.1)$ & $2079(66.0)$ & Reference & Reference \\
\hline
\end{tabular}

Current use, the drug use within 30 days before the index date; recent use, the drug use within 90 days, but not within 30 days, before the index date; past use, the drug use after the cohort entry, but not within 90 days before the index date.

${ }^{*}$ ORs of AKI for current/recent PPI users compared with past users were estimated using a conditional logistic regression model.

†Adjusted ORs were estimated by entering the potential confounders into the model.

use of NSAIDs or antibiotics among current PPI users. This analytical method was employed with reference to previous studies $^{32}{ }^{33}$ that have assessed risks of AKI associated with combined use of either antihypertensive drugs and NSAIDs. A crude incidence rate was calculated dividing the total number of cases in each exposure category by the category's person-years of follow-up. The Poisson distribution was used to determine $95 \%$ CIs for incidence rates.

\section{Sensitivity analyses}

We performed several sensitivity analyses to evaluate the robustness of the results. First, since it was considered that diagnosis of AKI might be delayed from the onset of the disease, we set time windows that the index date was 1 week before the diagnosis. Second, we followed the patients for up to only 120 days from the cohort entry, since most symptoms of drug-induced acute renal dysfunction were reported to occur early in the course of the therapy. ${ }^{34}$ Third, we explored whether including patients who had diagnostic codes of AIN (N10X or $\mathrm{N} 14 \mathrm{X}$ ) into cases altered the risk estimates. Fourth, we repeated the analyses without excluding patients who had diagnostic codes of renal diseases (online supplemental table 1) before the cohort entry; however, the patients who experienced AKI or dialysis before the entry were excluded from the analyses.

\section{Patient and public involvement}

Patients and the public were not involved in the study design and the conduct of the study.

\section{RESULTS}

\section{Study participants}

Among the patients eligible for this study, 58.3\% $(n=219082)$ had medical history more than 6 months before the first prescription of PPIs and did not have any history of renal disease before cohort entry (figure 2). The age on cohort entry was $45 \pm 13$ years (mean $\pm \mathrm{SD}$ ), and $44 \%$ were women. Patients were followed for a mean of $2.4 \pm 1.7$ years, generating a total of 519359 personyears of follow-up. There were 317 AKI cases during the follow-up period, yielding an overall crude incidence rate of 6.1/10 000 person-years (95\% CI, 5.5 to 6.8 ). We identified 3150 controls in this study cohort. The characteristics of the cases and controls are summarised in table 1. The types of PPIs prescribed at the last time before the index data were comparable between cases and controls. Lansoprazole was the most common PPI used in this population. The proportions of current users of nephrotoxic drugs, NSAIDs, penicillins, cephalosporins and fluoroquinolones in cases were significantly higher than those in controls.

Table 3 Effect of NSAID or antibiotic use on the risk of AKI among current PPI users

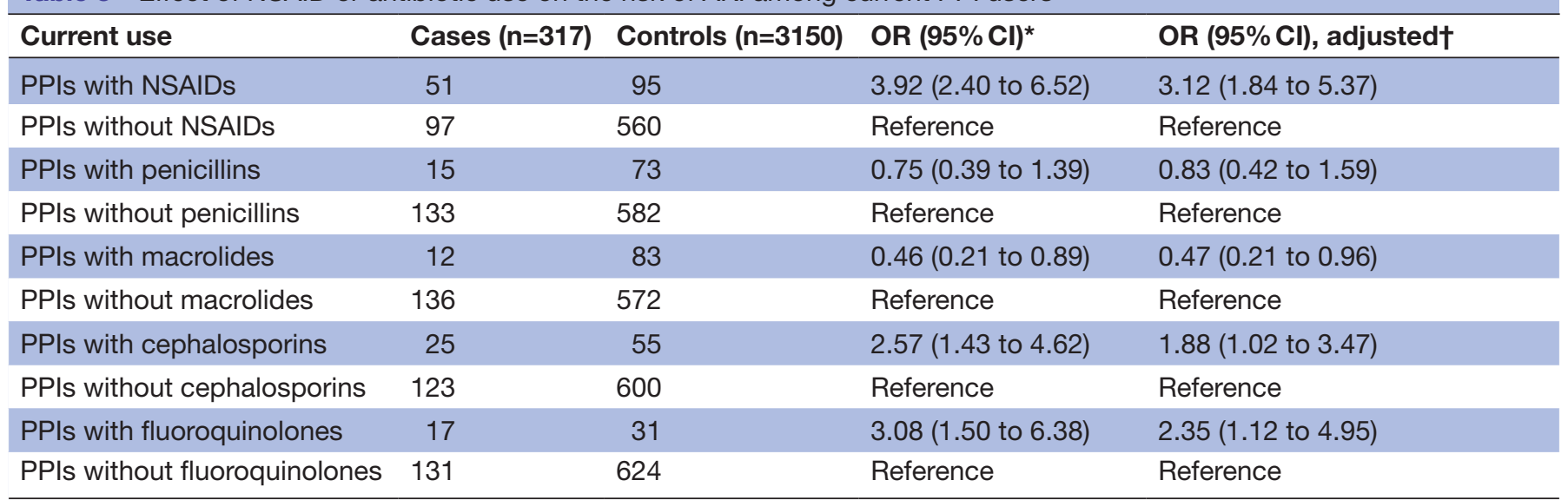

${ }^{*}$ ORs of AKI for current drug combinations compared with PPIs alone were estimated using the conditional logistic regression model.

†Adjusted ORs were estimated by entering the potential confounders into the model.

AKI, acute kidney injury; NSAIDs, non-steroidal anti-inflammatory drugs; PPIs, proton pump inhibitors. 
Table 4 Crude incidence rates of AKI

\begin{tabular}{|c|c|c|c|}
\hline Exposure & Number of cases & Person-year & Incidence rate per 10000 person-year $(95 \% \mathrm{Cl})$ \\
\hline Current use of PPIs & 148 & 78780 & 18.8 (15.9 to 22.1$)$ \\
\hline Recent use of PPls & 23 & 43399 & $5.3(3.4$ to 8.0$)$ \\
\hline \multicolumn{4}{|l|}{ Concomitant use } \\
\hline PPIs with NSAIDs & 51 & 13315 & 38.3 (28.5 to 50.4 ) \\
\hline PPIs with macrolides & 12 & 9904 & 12.1 (6.3 to 21.2) \\
\hline PPIs with cephalosporins & 25 & 5555 & $45.0(29.1$ to 66.4$)$ \\
\hline PPIs with fluoroquinolones & 17 & 3970 & 42.8 (24.9 to 68.6$)$ \\
\hline
\end{tabular}

Crude incidence rates were calculated by dividing the total number of cases in each exposure category by the category's person-years of follow-up. The Poisson distribution was used to determine 95\% Cls for incidence rates.

AKI, acute kidney injury; NSAIDs, non-steroidal anti-inflammatory drugs; PPIs, proton pump inhibitors.

\section{Effect of PPI use on the risk of AKI}

Initially, we assessed whether current use of PPIs was related to an increased risk of AKI (table 2). The estimated OR of AKI for current PPI users compared with past PPI users was 4.09 (95\% CI, 3.09 to 5.44). After adjusting for potential confounders (current use of nephrotoxic drugs and CCI), the estimated OR of AKI for current PPI users was 2.79 (95\% CI, 2.06 to 3.79). The effects of the recent use of PPIs compared with past use were not significant.

\section{Effect of NSAID or antibiotic use on the risk of AKI among current PPI users}

The characteristics of current PPI users in cases and controls are summarized in online supplemental table 4. Table 3 shows the summary of the primary analysis. The combined use of NSAIDs, cephalosporins and fluoroquinolones significantly increased the risks of AKI in current PPI users. The adjusted ORs for combined use of NSAIDs, cephalosporins and fluoroquinolones in current PPI users were 3.12 (95\% CI, 1.84 to 5.37), 1.88 (95\% CI, 1.02 to 3.47 ) and 2.35 (95\% CI, 1.12 to 4.95 ), respectively. In the sensitivity analyses, the significantly increased risks of AKI were still observed by concomitant use of NSAIDs or cephalosporins in current PPI users (online supplemental table 5). Additionally, the primary analysis showed that current use of PPIs with macrolides reduced the risk of AKI (the adjusted OR, 0.47; 95\% CI, 0.21 to 0.96 ) (table 3); however, the direction of effect was inconsistent in the sensitivity analyses (online supplemental table 5).

\section{Absolute incidence rate of AKI}

Table 4 shows the crude incidence rates of AKI per 10000 person-years in the study cohort. The estimated incidence rates of AKI for current, recent and past use of PPIs were 18.8 (95\% CI, 15.9 to 22.1 ), 5.3 (95\% CI, 3.4 to 8.0 ) and 3.7 (95\% CI, 3.1 to 4.3$)$, respectively. The incidence rates for concomitant use of NSAIDs, cephalosporins or fluoroquinolones with PPIs were substantially higher than those with other drug combinations or sole (incidence rate for current use of PPIs without NSAIDs or antibiotics, 15.3 (95\% CI, 12.1 to 19.1$)$ ).

\section{DISCUSSION}

We assessed the association between the concomitant use of PPIs with NSAIDs or antibiotics (penicillins, macrolides, cephalosporins and fluoroquinolones) on the risk of AKI. As the results of this study, we found that concomitant use of NSAIDs, cephalosporins or fluoroquinolones significantly increased the risk of AKI development in current PPI users. The significant effects of concomitant drugs were detected by several sensitivity analyses, and even when adjusting for potential confounders.

Since PPIs are widely prescribed for the prevention or treatment of peptic ulcer caused by NSAIDs, ${ }^{2}$ these two classes of medicines are often co-prescribed. Thus, it would be necessary to evaluate the effect of concomitant use of these drugs on the risk of AKI. There are two studies in which risks of AKI have been assessed when PPIs and NSAIDs had been concomitantly used; ${ }^{10} 13$ however, the findings have been limited. Svanström $e t a l^{10}$ have focused on the association between PPI use and incident AKI and evaluated the effect of concomitant use of NSAIDs as a subgroup analysis. However, the number of observed outcome events in the subgroup analysis was too small to have an adequate power to investigate the association between NSAID use and the risk of AKI. ${ }^{10}$ In the other study by Leonard et $a l^{13}$ patients' medication history before the study entry have not been evaluated; thus, the risk of AKI in PPI users could be underestimated and the result might be affected by selection bias. We conducted a nested-case control study within a cohort of PPI users who were first-time users and restarting users to reduce the effect of confounding by indication. Our findings indicated that concomitant use of NSAIDs with PPIs was associated with an increased risk of incident AKI, which were consistent in the sensitivity analyses. 
Penicillins, cephalosporins and fluoroquinolones are frequently prescribed antibiotics that are known to evoke $\mathrm{AKI}^{202435}$ and AIN. ${ }^{21} 36$ In additions, macrolides are suspected to increase the risk of AKI. ${ }^{22}{ }^{23}$ We hypothesised that these classes of antibiotics might affect the risk of incident AKI in PPI users. To prove this hypothesis, we evaluated the effect of concomitant use of antibiotics with PPIs. Although the present analyses were limited due to small number of cases examined, the results suggested that the concomitant use of cephalosporins or fluoroquinolones with PPIs was associated with increased risk of incident AKI. In contrast, penicillins and macrolides might not increase the AKI risk in PPI users. A previous study investigated the risk of AKI associated with antibiotics commonly prescribed in the community and showed a reduction in the incident rate ratio of AKI for macrolides $(0.79 ; 95 \%$ CI, 0.66 to 0.95$) .{ }^{20}$ Moreover, in our study, the results of the main analysis also suggested a reduction in the relative risk of AKI for current use of macrolides in PPI users. These results might represent the direct effect of macrolides on the development of AKI and the difference in severe systemic diseases between the macrolide users and the groups of other antibiotics or NSAID users. The validity of our results should be elucidated in future studies.

PPIs examined in this study ranged from classical types to a novel potassium-competitive acid blocker, vonoprazan. With a small number of vonoprazan users in our cohort, we could not find any significant difference in the AKI risks among types of PPIs. This result indicated that the inhibition of acid secretion is a possible causative factor to increase the risk of AKI. Given that the risk of adverse renal outcomes with vonoprazan use remained uncertain, a further pharmacovigilance study on the use of vonoprazan should be warranted.

Our study had significant limitations. First, the database used here did not include serum creatinine and glomerular filtration rate. Although previous reports have shown that the definition of AKI using the ICD-10 codes is specific for moderate-to-severe AKI, ${ }^{17}{ }^{37}$ the selection using this code limits the ability to evaluate the severity of AKI. It has been pointed out that measuring AKI using the ICD-10 codes underestimates the true risk of AKI. ${ }^{33}$ Second, patients' renal functions at baseline, a major risk factor for AKI, ${ }^{38}$ could not be evaluated. Third, the index date was usually behind the onset of AKI. The time delay from the onset of AKI to its diagnosis might result in misclassification of the exposure status and reverse causation. Nevertheless, both the primary and sensitivity analyses gave consistent results in the effect of current PPI use, suggesting that this limitation could be unlike to be critical. Fourth, we could not account for over-thecounter (OTC) use of the study drugs. However, PPIs and antibiotics of interest in this study have not been available as OTC drugs in Japan. Although NSAIDs were available as OTC drugs, we expected that long-term users of NSAIDs from the OTC were quite few in Japan. Fifth, the patients' medication adherence was uncertain. Sixth, we were unable to collect claims data of patients before their enrolment in the database. Some patients were possibly diagnosed with renal diseases and treated with PPIs before entry into the database. Seventh, residual confounding by unmeasured or imprecisely measured confounders may exist. Considering that subspecification of the exposure would limit the power of this study, we did not distinguish specific classes of nephrotoxic drugs and comorbidities. In addition, confounding by indications or confounding by contraindication are also possible. Eighth, the rarity of the outcome led to limited number of cases. This affected the precision of the estimates as shown by the wide CIs. Finally, generalisation of these results should be done with caution since the patients in this study were relatively younger than those in previous studies. ${ }^{3-10}$

In conclusion, concomitant use of NSAIDs with PPIs significantly increased the risk of AKI. Thus, physicians should be aware that patients who concomitantly use PPIs and NSAIDs would have a pronounced risk for AKI. Furthermore, our findings suggested that concomitant use of cephalosporins or fluoroquinolones with PPIs was associated with increased risks of AKI. These results motivate the need for further studies to confirm the associations and investigate the biological mechanisms.

Contributors KIk, SN and KMo were responsible for developing the study concept and design. KIk and SN performed the statistical analysis, wrote the initial draft of the manuscript and revised the manuscript. KMo and AY contributed to the data collection. KIk, SN, KMo, AY and KMa contributed to the interpretation of the data. KIt, YS, SI, TN contributed to data validation. KIt, YS, SI, TN and KMa critically reviewed the manuscript. All authors read and approved the final version of the manuscript.

Funding This study was supported by a grant-in-aid for Scientific Research (KAKENHI) from the Japan Society for the Promotion of Science (grant JP18K06783).

Competing interests None declared.

Patient consent for publication Not required.

Ethics approval The study protocol was approved by the Kyoto University Hospital Ethics Committee (R2262).

Provenance and peer review Not commissioned; externally peer reviewed.

Data availability statement The data that support the findings of this study are available from JMDC Inc. (https://www.jmdc.co.jp) but restrictions apply to the availability of these data, which were used under license for the current study and so are not publicly available.

Supplemental material This content has been supplied by the author(s). It has not been vetted by BMJ Publishing Group Limited (BMJ) and may not have been peer-reviewed. Any opinions or recommendations discussed are solely those of the author(s) and are not endorsed by BMJ. BMJ disclaims all liability and responsibility arising from any reliance placed on the content. Where the content includes any translated material, BMJ does not warrant the accuracy and reliability of the translations (including but not limited to local regulations, clinical guidelines, terminology, drug names and drug dosages), and is not responsible for any error and/or omissions arising from translation and adaptation or otherwise.

Open access This is an open access article distributed in accordance with the Creative Commons Attribution Non Commercial (CC BY-NC 4.0) license, which permits others to distribute, remix, adapt, build upon this work non-commercially, and license their derivative works on different terms, provided the original work is properly cited, appropriate credit is given, any changes made indicated, and the use is non-commercial. See: http://creativecommons.org/licenses/by-nc/4.0/.

ORCID iDs

Shunsaku Nakagawa http://orcid.org/0000-0002-1285-122X

Atsushi Yonezawa http://orcid.org/0000-0002-8057-6768 


\section{REFERENCES}

1 Nochaiwong S, Ruengorn C, Awiphan R, et al. The association between proton pump inhibitor use and the risk of adverse kidney outcomes: a systematic review and meta-analysis. Nephrol Dial Transplant 2018;33:331-42.

2 Freedberg DE, Kim LS, Yang Y-X. The risks and benefits of longterm use of proton pump inhibitors: expert review and best practice advice from the American gastroenterological association. Gastroenterology 2017;152:706-15.

3 Lee J, Mark RG, Celi LA, et al. Proton pump inhibitors are not associated with acute kidney injury in critical illness. J Clin Pharmacol 2016;56:1500-6.

4 Lazarus B, Chen Y, Wilson FP, et al. Proton pump inhibitor use and the risk of chronic kidney disease. JAMA Intern Med 2016;176:238-46.

5 Xie Y, Bowe B, Li T, et al. Proton pump inhibitors and risk of incident CKD and progression to ESRD. J Am Soc Nephrol 2016;27:3153-63.

6 Klatte DCF, Gasparini A, Xu H, et al. Association between proton pump inhibitor use and risk of progression of chronic kidney disease. Gastroenterology 2017;153:702-10.

7 Hart E, Dunn TE, Feuerstein S, et al. Proton pump inhibitors and risk of acute and chronic kidney disease: a retrospective cohort study. Pharmacotherapy 2019;39:443-53.

8 Klepser DG, Collier DS, Cochran GL. Proton pump inhibitors and acute kidney injury: a nested case-control study. BMC Nephrol 2013;14:150.

9 Antoniou T, Macdonald EM, Hollands S, et al. Proton pump inhibitors and the risk of acute kidney injury in older patients: a populationbased cohort study. CMAJ Open 2015;3:E166-71.

10 Svanström H, Lund M, Melbye M, et al. Use of proton pump inhibitors and the risk of acute kidney injury among patients with rheumatoid arthritis: cohort study. Drug Saf 2018;41:817-26.

11 Seethapathy H, Zhao S, Chute DF, et al. The incidence, causes, and risk factors of acute kidney injury in patients receiving immune checkpoint inhibitors. Clin J Am Soc Nephrol 2019;14:1692-700.

12 Cortazar FB, Kibbelaar ZA, Glezerman IG, et al. Clinical features and outcomes of immune checkpoint inhibitor-associated AKI: a multicenter study. J Am Soc Nephrol 2020;31:435-46.

13 Leonard CE, Freeman CP, Newcomb CW, et al. Proton pump inhibitors and traditional nonsteroidal anti-inflammatory drugs and the risk of acute interstitial nephritis and acute kidney injury. Pharmacoepidemiol Drug Saf 2012;21:1155-72.

14 Tanaka S, Seto K, Kawakami K. Pharmacoepidemiology in Japan: medical databases and research achievements. J Pharm Health Care Sci 2015;1:16.

15 Mushiroda T, Takahashi Y, Onuma T, et al. Association of HLA- $A * 31: 01$ screening with the incidence of carbamazepineinduced cutaneous adverse reactions in a Japanese population. JAMA Neurol 2018;75:842-9.

16 Momo K, Takagi A, Miyaji A, et al. Assessment of statin-induced interstitial pneumonia in patients treated for hyperlipidemia using a health insurance claims database in Japan. Pulm Pharmacol Ther 2018;50:88-92.

17 Hwang YJ, Shariff SZ, Gandhi S, et al. Validity of the International classification of diseases, tenth revision code for acute kidney injury in elderly patients at presentation to the emergency department and at hospital admission. BMJ Open 2012;2:e001821.

18 Lafrance J-P, Miller DR. Selective and non-selective non-steroidal anti-inflammatory drugs and the risk of acute kidney injury. Pharmacoepidemiol Drug Saf 2009;18:923-31.
19 Ungprasert P, Cheungpasitporn W, Crowson CS, et al. Individual non-steroidal anti-inflammatory drugs and risk of acute kidney injury: a systematic review and meta-analysis of observational studies. Eur J Intern Med 2015;26:285-91.

20 Rennie TJW, De Souza N, Donnan PT, et al. Risk of acute kidney injury following community prescription of antibiotics: self-controlled case series. Nephrol Dial Transplant 2019;34:1910-6.

21 Muriithi AK, Leung N, Valeri AM, et al. Biopsy-proven acute interstitial nephritis, 1993-2011: a case series. Am J Kidney Dis 2014;64:558-66.

22 Woodruff AE, Meaney CJ, Hansen EA, et al. Azithromycin-Induced, biopsy-proven acute interstitial nephritis in an adult successfully treated with low-dose corticosteroids. Pharmacotherapy 2015;35:e169-74.

23 Li DQ, Kim R, McArthur E, et al. Risk of adverse events among older adults following co-prescription of clarithromycin and statins not metabolized by cytochrome P450 3A4. CMAJ 2015;187:174-80.

24 Bird ST, Etminan M, Brophy JM, et al. Risk of acute kidney injury associated with the use of fluoroquinolones. CMAJ 2013;185:E475-82

25 Blank M-L, Parkin L, Paul C, et al. A nationwide nested case-control study indicates an increased risk of acute interstitial nephritis with proton pump inhibitor use. Kidney Int 2014;86:837-44.

26 Lai T-S, Wang C-Y, Pan S-C, et al. Risk of developing severe sepsis after acute kidney injury: a population-based cohort study. Crit Care 2013;17:R231.

27 Koulouridis I, Price LL, Madias NE, et al. Hospital-acquired acute kidney injury and hospital readmission: a cohort study. Am J Kidney Dis 2015;65:275-82.

28 Ozrazgat-Baslanti T, Thottakkara P, Huber M, et al. Acute and chronic kidney disease and cardiovascular mortality after major surgery. Ann Surg 2016;264:987-96.

29 Sundararajan V, Henderson T, Perry C, et al. New ICD-10 version of the Charlson comorbidity index predicted in-hospital mortality. J Clin Epidemiol 2004;57:1288-94.

30 Quan H, Sundararajan V, Halfon P, et al. Coding algorithms for defining comorbidities in ICD-9-CM and ICD-10 administrative data. Med Care 2005;43:1130-9.

31 Breslow NE. Statistics in epidemiology: the case-control study. J Am Stat Assoc 1996;91:14-28.

32 Lapi F, Azoulay L, Yin H, et al. Concurrent use of diuretics, angiotensin converting enzyme inhibitors, and angiotensin receptor blockers with non-steroidal anti-inflammatory drugs and risk of acute kidney injury: nested case-control study. BMJ 2013;346:e8525.

33 Dreischulte T, Morales DR, Bell S, et al. Combined use of nonsteroidal anti-inflammatory drugs with diuretics and/or reninangiotensin system inhibitors in the community increases the risk of acute kidney injury. Kidney Int 2015;88:396-403.

34 Sierra F, Suarez M, Rey M, et al. Systematic review: proton pump inhibitor-associated acute interstitial nephritis. Aliment Pharmacol Ther 2007;26:545-53.

35 Bellomo R, Kellum JA, Ronco C. Acute kidney injury. Lancet 2012;380:756-66.

36 Pannu N, Nadim MK. An overview of drug-induced acute kidney injury. Crit Care Med 2008;36:S216-23.

37 Waikar SS, Wald R, Chertow GM, et al. Validity of international classification of diseases, ninth revision, clinical modification codes for acute renal failure. J Am Soc Nephrol 2006;17:1688-94.

38 Grams ME, Astor BC, Bash LD, et al. Albuminuria and estimated glomerular filtration rate independently associate with acute kidney injury. J Am Soc Nephrol 2010;21:1757-64. 